\title{
Dos formas distintas de investigar el problema del paro en los albores de la encuesta social. El juicio público frente a la taxonomía
}

\author{
MARIA GÓMEZ GARRIDO \\ Instituto Universitario Europeo \\ Maria.Gomez@IUE.it
}

Recepción: diciembre 2004

Aceptación: abril 2005

El paro ha sido uno de los fenómenos de mayor calado en las sociedades occidentales de los últimos treinta años. Sus desorbitadas cifras durante la década de los ochenta (de las que España, como sabemos, batió todos los récords) lo convirtieron en la preocupación número uno de las encuestas de opinión, y el objetivo primordial de las políticas económicas y sociales.

Cuando hablamos del paro, parece que nos referimos siempre a una misma cosa. Tras dos siglos de presencia en nuestros vocabularios, el paro se ha convertido en un concepto de referencia compartida. No sólo podemos contar en millones las obras que hoy en día analizan sus cifras comparando países, regiones localidades; sino que también se cuentan en miles las que lo estudian en el pasado. Desde economistas que han tratado de explicar la evolución del mismo en función de distintas variables, a historiadores sociales lo han presentado como la causa directa del estallido de movilizaciones sociales.

Todas estas obras suelen tener como fuente un mismo instrumento: las estadísticas de paro. Es este tipo de documento el que otorga forma y, casi podríamos decir, el que constituye el paro en un «hecho». En nuestras sociedades actuales, las cifras suministradas por organismos investidos de autoridad, como Naciones Unidas o la Oficina Internacional del Trabajo, suelen ser recibidas como una fotografía exacta de la realidad. Este hábito nos lleva a proyectar en nuestra mirada hacia el pasado la búsqueda de datos equivalentes sobre los que apoyar un juicio certero.

Sin embargo, al estudiar un aspecto cualquiera de la vida social, como es el caso del paro, rápidamente salta a la vista las múltiples dimensiones que dicho concepto ha adquirido a lo largo del tiempo. Pues pese a que con el término paro se ha pretendido aludir siempre a la falta de trabajo, la identificación concreta del parado ha experimentado una importante variación en cada contexto histórico- 
político. Esto, que puede ser visto como problema, ha sido aprovechado por toda una serie de obras recientes, que tratan precisamente de dar cuenta de la historia de las categorías estadísticas y sociales y de vincular su significado a cada contexto político y social.

En este sentido, al emprender la historia de una categoría estadística concreta, resulta útil establecer una distinción analítica entre concepto y categoría. El concepto hace referencia al campo semántico en un sentido amplio. Es el caso de los debates en torno a la falta de trabajo que contribuyeron a la creación de un nuevo problema en las últimas décadas del siglo diecinueve. La emergencia de este nuevo concepto traerá un cambio en las formas de entender la pobreza con relación al trabajo.

No obstante, la formación de un nuevo concepto no supone la identificación inmediata de situaciones que puedan establecerse sobre un mismo plano de equivalencia. Esto tiene lugar sólo a través de un proceso de categorización. Así pues, la categoría es la materialización de un concepto a través de instrumentos como la estadística y el derecho (Zimmermann 2001: 9) ${ }^{1}$. La importancia de ese proceso de materialización de un concepto, hace que la historia de una categoría como el paro esté marcada no sólo por acontecimientos generales de la vida social, como puede ser la historia del movimiento obrero o la dimensión de género, sino también por la propia historia de la estadística como disciplina, y concretamente, por la historia de la encuesta como forma de conocimiento de lo social. Este artículo se centrará en este segundo aspecto.

Son habituales las quejas de la ausencia de «buenas» estadísticas por parte de quien, por ejemplo, quiere estudiar un fenómeno como el paro en algún período histórico. Por «buenas» se da a entender estadísticas que sigan procedimientos de sistematización semejantes a los actuales. Tal visión asume que los sucesivos cuadros estadísticos han permitido un mejor acercamiento a la realidad a través de un aparato más sofisticado de observación, como si fuese el propio aparato el que nos permitiera conocer el fenómeno.

Pero esta actitud casi inconsciente cambia cuando se reflexiona sobre la propia historia de los métodos de investigación y los aparatos de representación

${ }^{1}$ La corriente reflexiva sobre la historia de las categorías es en cierto sentido heredera de la Breggriffsgeschichte o historia de los conceptos. Sin embargo, la historia de las categorías afina aún más la lente de observación en cuanto que no sólo procede a estudiar la historia de un fenómeno, un grupo social o una institución, sino que también da cuenta de la condición historica de las categorías de análisis y representación de dicho objeto (Werner et Zimmermann 2003). Cabe pensar, sin embargo, si esta diferencia tan enfatizada por la propia historiografía no responde también a los respectivos temas de investigación. Pues la historia de las categorías se ha centrado en el período en el que las ciencias sociales han adquirido un papel fundamental, mientras que la historia de los conceptos ha prestado atención a períodos anteriores. En el primer caso nos encontraríamos ante un grado mayor de complejidad en los fenómenos estudiados (por la multiplicación de las representaciones), y por tanto, el nivel de observación pertinente variaría. Por otra parte, existe una diferencia teórica más importante y es que la historia de las categorías adopta el enfoque del pragmatismo, por lo que presta atención a las práticas de los actores para, a partir de ellas, comprender el significado de su lenguaje, y no al revés. 
del mundo social. A través de la misma resulta claro hasta qué punto los procedimientos por los que se puede conocer un fenómeno no han sido (ni son) algo evidente e inmediato.

Pues la sociología nace de la mano de la estadística como parte de un esfuerzo secular por dotarse de una representación estable del orden social. De este esfuerzo emergerá una nueva forma de gobernar que caracteriza lo que conocemos como modernidad. Sin embargo, desde el nacimiento de estas dos disciplinas hasta su aceptación como instrumentos clave en la política tiene lugar un largo proceso no exento de conflictos y vacilaciones. La fundación del Instituto Internacional de Estadística en 1885 abre un largo período que se extiende hasta los años cuarenta y cincuenta del siglo veinte en el que no habrá un consenso en torno a la práctica de la estadística, ni los técnicos gozarán de una posición institucional reconocida en todos los países ${ }^{2}$.

El nacimiento de la sociología y la estadística tiene lugar en el contexto de fuerte conflicto político, al que se alude en la época como cuestión social. Colectivos muy diferentes reclamarán la necesidad de un conocimiento científico de tales problemas. Ahora bien, la idea de ciencia, como la posibilidad de establecer la veracidad sobre un fenómeno social, presenta dos vertientes a finales del siglo diecinueve:

La primera, progresivamente asentada en el imaginario colectivo, presenta la ciencia como la combinación de aparato técnico y racionalidad humana, a través de la cual se puede descubrir la naturaleza y mecanismos de los fenómenos sociales. En esta imagen, el experto descubre una verdad oculta, operando a través de un distanciamiento de las visiones del común. Esta idea de objetividad, que Porter ha denominado mecánica, llevará incluso al objetivo de obtener un distanciamiento de la propia subjetividad del sujeto que observa, considerada excesivamente débil y cambiante (Porter 1995: 76). Es así como se introducirá progresivamente un lenguaje matemático que dará lugar con el tiempo a la formación de la estadística matemática.

La segunda, de la que participan las organizaciones obreras, pero también algunos socialistas y reformadores sociales, entiende la ciencia de lo social como

${ }^{2}$ Ruyesmeyer y Skocpol (1996) han planteado la hipotesis de que los orígenes de la política social, es decir, de las reformas legislativas hacia las clases trabajadoras derivadas de conceptos propios de una serie de discplinas emergentes como la estadística y la sociología, depende directamente de la capacidad organizativa de los grupos de reformadores, sociologos y estadísticos, que, en la medida que lograron la institucionalización de sus respectivas disciplinas, pudieron imponer a finales del siglo diecinueve una nueva forma de representar las cosas. Sin duda, la idea de que a mayor grado de automía, mayor poder tendrá el técnico o estadístico en la forma de representar el mundo social parece una intuición cargada de sentido común. Sin embargo, es difícil aceptar esta trasposición del modelo de acción colectiva de Tilly, y de los propios trabajos de Theda Skocpol sobre el Estado, al caso concreto de los científicos sociales, tomados como corporación o grupo social con intereses propios. En los albores de la encuesta social, no habrá consenso entre los propios «investigadores» sobre los procedimientos de obtención de datos del mundo social. De tal manera que el proceso de definición de las prácticas de cada disciplina será inseparable de procesos políticos más amplios. 
el conocimiento de una realidad ocultada por la ideología o los discursos oficiales. La ciencia de lo social es principalmente el acercamiento a un sujeto hasta ahora enmudecido por la política oficial: la clase obrera. Ésta emerge así a finales del siglo como representante del «pueblo», de esa «realidad» desconocida oficialmente.

En este artículo me propongo analizar algunos de los primeros documentos de investigación social que surgen en este período, y que directa o indirectamente tratan la cuestión de la falta de trabajo. El contraste entre unos y otros pretende mostrar no tanto la dificultad en identificar a los parados, como las diversas formas de construir objetividad respecto a la situación. Así pues, esta perspectiva entronca con las reflexiones recientes del campo de la filosofía de la ciencia que centran su atención no ya en la historia de un hecho empírico (el paro) sino más bien en la historia de las distintas maneras de establecer lo empírico, es decir, los distintos procedimientos aceptados como científicos para describir la realidad (Chandler 1991: 282-283). A través de una comparación entre la encuesta anglosajona, y las informaciones españolas, el texto analiza las maneras en que se lidió con la controvertida cuestión de la representatividad en cada caso.

\section{EL NACIMIENTO DEL PARO}

Puede resultar irritante oír que el paro es un concepto «inventado» a finales del siglo diecinueve (Salais et al. 1986). Reflexiones sobre el vagabundeo, o sobre aquél sin «oficio ni beneficio conocido» datan al menos del siglo dieciséis. Y a lo largo de los siglos dieciocho y diecinueve, se forma en varias ciudades europeas un mercado a partir de una masa creciente de personas que vagan de un trabajo a otro. No obstante, como ha señalado Christian Topalov, estas masas de obreros sin trabajo «no eran parados, ya que en ese momento se carecía de un concepto con el que representar dicha situación» (1994: 24). Dicho concepto nacerá con el cambio de siglo, en un momento en el que, por otra parte, no parece que se produzca un aumento del número de obreros sin trabajo ${ }^{3}$, sino que más bien se da una serie de condiciones que permitirán representar la falta de trabajo como paro.

La pobreza y el trabajo habían conformado dos ejes fundamentales en el debate sobre las formas de vida de las clases populares de la Inglaterra victoriana. Médicos, moralistas e higienistas se unieron en una preocupación por la supuesta degeneración de los hábitos de aquellos grupos sociales que ellos tomaron como objeto de análisis: los pobres.

Pero hacia finales de ese período, la cuestión de la pobreza y las formas de vida de las clases pobres adquirirán un sentido totalmente nuevo a través de la

${ }^{3}$ T. S. Asthon ha investigado las cifras de obreros sin trabajo elaboradas por los sindicatos británicos desde los años veinte del siglo diecinueve y observa un decrecimiento en los años ochenta del mismo siglo. Esto le lleva a concluir que la emergencia del término en esas mismas fechas no es el resultado de una incidencia mayor del desempleo ( $c f$. Himmerlfab 1991: 43). 
denuncia llevada a cabo por el movimiento obrero, y los objetivos democratizadores de socialistas y republicanos. La Crítica de la Economía Política de Marx forma parte de esta nueva representación de los problemas, a la que contribuye especialmente: la pobreza empieza a verse como el resultado de las formas de organización de la sociedad entera, separando así la situación de los pobres de sus supuestas características individuales.

La sensación de crisis a finales del siglo diecinueve unirá a un número creciente de voces críticas frente a las propuestas liberales, y a favor de la intervención pública como solución a la falta de trabajo. Unos y otros, desde socialistas y solidaristas a reformistas y liberales, así como la mayor parte de las asociaciones obreras, plantearán la necesidad del conocimiento científico del fenómeno como única vía de enjuiciar la situación.

En el seno del movimiento reformista, la circulación de ideas facilitada por el creciente numero de conferencias y exposiciones bajo la temática de la reforma social dará lugar a una cuasi comunidad epistémica que genera una representación común de los problemas sociales y de las formas posibles de tratarlos. Es en el seno de este grupo o red internacional donde nacerá el concepto de parado (Lecerf 1989, Topalov 1994) ${ }^{4}$.

El nacimiento del concepto de paro trae consigo una transformación de los campos de la pobreza y la asistencia. La falta de trabajo recibe a partir de ese momento un nombre propio, que trata de representar aquella situación que se produce más allá de la voluntad del sujeto, que se debe a factores externos a él. Con el fin de atajar dicho problema se plantea en todos los foros la necesidad de recopilar estadísticas que ofrezcan un conocimiento exacto de las dimensiones y la distribución del paro.

Pero las prácticas de una ciencia de lo social se encuentran en ciernes en esos momentos. Así, la falta de trabajo, que constituye un eje del conflicto social de finales del siglo diecinueve, va a ser enjuiciada a través de muy distintas formas de investigación. La elaboración de las mismas no será una tarea sencilla por otro motivo añadido: El paro es un concepto de nuevo cuño, para el que aún no existe una política que lo dé forma. Este hecho lo diferencia, por ejemplo, de las estadísticas sobre población asistida elaboradas a partir de los establecimientos de beneficencia. La constitución del paro como categoría va a estar relacionada con los sujetos llamados a recitar dicha situación. En algunos casos serán las propias asociaciones obreras las que, apoyándose en su experiencia de fondos de solidaridad frente a unas condiciones de trabajo duras e inestables, den forma a la categoría del paro. En otros casos el médico, sociólogo o técnico de estadística, construirán taxonomías concretas a las que traducir distintas situaciones observadas. Las implicaciones políticas de una u otra opción serán diferentes.

${ }^{4}$ Sobre la importancia en general de los congresos y exposiciones internacionales como lugares de circulación de ideas y nuevos espacios de representación se puede leer el número 7 de la revista Mil Neuf Cent, 1989. 


\section{LA ENCUESTA ANGLOSAJONA}

Por paradójico que pueda resultar, es en el mundo liberal anglosajón donde, en los años treinta del siglo diecinueve, surge un interés por lo que será el antecedente de la encuesta social. Su nacimiento es el resultado de una nueva mirada, que se caracteriza por dos aspectos:

En primer lugar, lo que podríamos definir como un sentimiento de sorpresa o desconcierto (puzzlement). Si los filósofos del siglo dieciocho experimentaron ese sentimiento de desconcierto y curiosidad por los habitantes de otros países, los reformadores del siglo diecinueve sienten esta curiosidad por personas de su propio país que son, sin embargo, diferentes a ellos mismos (Bulmer y Sklar 1991).

Tal sentimiento se desata especialmente en el ámbito urbano, donde, bajo la niebla y el humo de las nuevas fábricas, surge una masa creciente de trabajadores cuya identidad se oscurece al carecer de los canales de reconocimiento que existían en el mundo rural, y que insertaban al sujeto en un grupo concreto, definiendo las reglas de sus relaciones inter y extragrupales ${ }^{5}$. En este contexto, las clases medias y altas de la Inglaterra victoriana desarrollan una visión ambientalista que idealiza el mundo rural. Sus habitantes son alabados por «la sobriedad, la frugalidad y la laboriosidad», contrastando con la situación de la ciudad en la que «la insalubridad y los hacinamientos conducían a la deterioración moral, y a la pérdida de los sentimientos delicados» (Journal of the Statistical Society of London 1842, cf. Cullen 1975: 136).

Pero más allá de las consecuencias de la vida anónima de la ciudad, y las preocupaciones moralistas de los victorianos, este sentimiento de desconcierto tuvo como origen también los intentos de ciudadanos ilustrados por ver a los habitantes de su país como iguales. De esta visión sobre la universalidad humana surgirá una lucha incesante por reducir la desigualdad, pero inevitablemente, surgirá también una tendencia a eliminar la diferencia. Es este punto de partida el que está en la base de la nueva ciencia social. De tal manera que la sociología empírica anglosajona hunde sus raíces en estas reflexiones que jalonan el período victoriano y que abren el camino a los fabianos y a la nueva generación de reformadores sociales.

En segundo lugar, la encuesta nace no sólo de la curiosidad, sino de un sentimiento de sospecha. Higienistas y utilitaristas inaugurarán esta nueva mirada de un observador externo que intenta descubrir una verdad escondida. Ya en 1789, Bentham proponía enviar bajo la jurisdicción de la parroquia local no sólo a aquellos que solicitaran ayuda, sino además a todos aquellos «sospechosos de ser pobres». Para poder identificar este último grupo, Bentham propuso la elaboración de un registro universal de la población con sus direcciones y ocupaciones detalladas (Himmelfarb [1983]1988: 97-105). Las soluciones propuestas

${ }^{5}$ El tema del anonimato en la ciudad será un eje fundamental de la naciente sociología. Uno de los pensadores que desarrolló esta cuestion con mayor originalidad como parte de sus reflexiones sobre la vida moderna es sin duda George Simmel. Por su parte, Halbawchs también reflexionó sobre los contrastes entre el mundo rural y urbano en los términos aquí presentados. 
por el padre del utilitarismo horrorizaron a muchos de sus contemporáneos. No obstante, la idea de interrogar con objeto de diferenciar y distinguir a grupos no visibles fundó las bases de una nueva práctica a través de la cual se podía emitir un juicio sobre una situación.

Desde principios del siglo diecinueve, la observación acompañada de interrogatorios a autoridades locales será una práctica corriente de los comités parlamentarios o selected committees. La evidencia presentada por estos comités servía como material de consenso previo a la aprobación de una nueva ley. La obsesión victoriana por la cuestión de la pobreza dio lugar a una incesante actividad de estos comités que trataban de observar con detalle las vidas de los pobres de distintas regiones con vistas a proponer nuevas regulaciones. Dichos comités estaban formados por "personalidades autorizadas", normalmente parlamentarios, miembros del clero, y economistas o médicos. (Himmelfarb 1988: 181-184; Bulmer 1985; Mash 1985). Aunque los informes se escribían con un estilo impresionista, son un primer paso que anticipa la idea de la necesidad de observación y análisis como requisito de la regulación del mundo social.

Como parte de este impulso por conocer un mundo oscuro y desconocido (el de las clases trabajadoras) y animados por la fe en las posibilidades de la naciente ciencia de lo social, se fundan a lo largo de la década de los treinta y cuarenta del siglo diecinueve numerosas sociedades estadísticas en ciudades como Manchester, Birmingham, o Londres. Estas sociedades promueven la realización de numerosas investigaciones lanzadas en el contexto local, y muchos de sus miembros colaboran también con los comités parlamentarios. El dinamismo en la actividad de estas sociedades ha llevado a hablar del movimiento estadístico o el movimiento de la encuesta (statistical o survey movement) ${ }^{6}$.

De él formarían parte también otra serie de asociaciones fundadas hacia mediados de siglo con el fin de promover líneas de actuación hacia las clases pobres que recogieran estas nuevas ideas de ciencia de lo social. Entre éstas se encuentran la National Association for the Promotion of Social Science), fundada en 1857 y de la que surgirá la Charity Organisation Society en 1861. Estas asociaciones están integradas por algunos industriales y hombres de negocios, así como por los nuevos profesionales de las clases medias emergentes (médicos, profesores de universidad o abogados), a los que se sumarán un número creciente de mujeres a mediados de siglo ${ }^{7}$. Así pues, estas sociedades han sido interpretadas también como un acto de distinción de las clases medias, frente a las clases altas y a los trabajadores. Por otra parte, las prácticas de investigación y

- Dentro de esta prehistoria de la sociología británica cabe sin duda señalar los relatos de Henry Mayhew, un testimonio muy ilustrativo de esa curiosidad de las clases medias del diecinueve por los «submundos» de la ciudad. El carácter periodístico de los mismos, sin embargo, los aleja de la temática de este artículo.

${ }^{7}$ Resulta muy interesante la interpretación de Yeo sobre la acción de estas mujeres de clase media. Su incorporación a una actividad aún fuertemente marcada por la lógica de la acción caritativa sería una manera de poder pasar a la acción pública preservando su «decencia». Esto sólo podía hacerse trasponiendo su rol de servicio en la familia a la esfera pública, y actuar así como «madres sociales» de los pobres (Yeo 1991: 54). 
promoción de políticas llevadas a cabo por estas sociedades constituyen una acción encaminada a solucionar el peligro potencial que presentaba el distanciamiento identitario de las clases bajas, el cual que venía acompañado por su proceso de segregación en determinados barrios de la ciudad (Jones 1971: 241357 y Jones 1981: 181-189).

Las encuestas e investigaciones realizadas por estas sociedades no son un antecedente de la estadística como disciplina. No hay ninguna matematización en las mismas, ni tampoco un interés en tal formalización. Los artículos publicados en el Journal of the Royal Statistical Society apenas entrarán en cuestiones metodológicas propias a la disciplina hasta los años treinta del siglo veinte (McKenzie 1981: 8-14) ${ }^{8}$. No obstante, el movimiento estadístico tiene una gran importancia en cuanto a que su presencia y actividad sentará las bases de una nueva forma de tratar lo social. Figuras clave en el mismo, como Edwin Chadwick o William Farr ${ }^{9}$ marcan ese punto de tránsito hacia las nuevas formas de gobernar.

\subsection{La encuesta de Booth sobre la pobreza: «Labour and Life of the People in London»}

Apoyándose en todo este bagaje, Charles Booth, presidente de la Royal Statistical Society, emprende en 1885 una investigación financiada por su propio presupuesto que se convertirá en un clásico en la historia de la encuesta y los estudios sobre la pobreza. El documento de Booth se sitúa en unos años de arduo debate sobre la situación de las clases pobres en Inglaterra, y sobre el problema de la falta de trabajo. Es éste un debate que tiene como trasfondo el proceso descrito arriba de la preocupación de las clases medias ante el progresivo distanciamiento del «pueblo» respecto a las normas establecidas, la religión y la fidelidad a los superiores.

Este hombre de negocios, que había sido una figura activa en los debates parlamentarios de años anteriores, tendrá un objetivo claro en su nueva empresa: estudiar las clases pobres, distinguiendo proporciones y relaciones. Es decir, salir de las «historias de caso», que tanto le irritaban porque daban «la impresión de

${ }^{8}$ Las primeras discusiones propiamente metodológicas se inauguran con la propuesta de Arthur Bowley de introducir métodos de muestreo para las encuestas y obtención de datos. La pobreza (1915) y el paro (1924) son precisamente las cuestiones sobre las que Bowley probará dichos métodos (Camarero 2001; Arribas 2002). Será de nuevo el paro el que funde la base de la primera encuesta por muestreo aleatorio, pero ya en los Estados Unidos del New Deal (Didier 2001).

9 Sir Edwin Chadwick (1800-1890), colaborador de Bentham, participó en las comisiones reales para reforma de las leyes de pobres en 1832. Entre 1848 y 1854 , ya en el Board of Health, promovió una reforma de la administración de la sanidad pública por la que el derecho a la asistencia vendrá condicionado por la pertenencia a la localidad, y administrado por las parroquias. William Farr (1807-1883), médico vinculado al Movimiento por la Salud Pública, fue nombrado director del General Register Office en 1837, momento que marca un cambio en esta institución desde la que se promoverán diversas investigaciones sobre la relación entre pobreza y morbilidad. (Desrosières 1993: 184-186). Su estudio sobre la mortalidad ocupacional servirá de base para la estadística de clases sociales del Register elaborada a principios del siglo veinte (Bulmer 1985). 
que todos los trabajadores son pobres» (Topalov 1991: 8). Booth se lanza así a la elaboración de una mastodóntica investigación de la zona este de Londres, que sería publicada después bajo el título Labour and Life of the People in London.

El objetivo fundamental de la encuesta de Booth consistirá en establecer una nítida taxonomía que le permita distinguir «sus pobres» del residuo, es decir, de los muy pobres, de los casos perdidos. Para ello se esfuerza en observar con detenimiento las características de estos grupos, sus hábitos, sus creencias, y poder «representar toda esta información en proporciones y relaciones».

\section{El informante privilegiado}

Desde un primer momento, Booth rechaza la idea de una encuesta directa a los sujetos a estudiar. Siguiendo la práctica de los selected committees y de las statistical organisations, las preguntas van dirigidas a uno o varios informantes privilegiados, es decir, personas, cuya autoridad local o posición institucional los hacía «representantes» de la zona estudiada (Mash 1985). En otras palabras, personas que se consideraba que podían transmitir un conocimiento justo de los grupos sociales a estudiar.

Para su investigación sobre la zona este de Londres, Booth se apoyó en los Visitadores de Escuelas Públicas (School Board Visitors) ${ }^{10}$. Booth justificaba la elección de los visitadores como informantes en razón de su contacto frecuente y control de la población a estudiar:

«Los Visitadores de Escuela están encargados de visitar casa por casa; todas y cada una de las casas de cada calle están en sus libros, y en ellos anotan detalles sobre cada familia con niños en edad de asistir a la escuela, incluyendo la ocupación del cabeza de familia. La mayor parte de los visitadores han estado trabajando en el mismo distrito durante varios años, y por tanto tienen un conocimiento extensivo de la gente (people). Entre sus obligaciones está el conocer lo más posible a los recién llegados a sus distritos. Tienen un contacto diario con todos ellos, y por esto mismo un conocimiento considerable de los padres de los niños más pobres, así como de las condiciones en las que viven» (Poverty Series I, 5 cf. Pfautz 1967: 49).

Los largos cuestionarios realizados a los visitadores, fueron completados con algunas entrevistas a los maestros, y con datos del censo. Sólo en la última fase de la investigación, el propio Booth y algunos de sus colaboradores se pasearon por la zona, e incluso pernoctaron en algunas de las casas de aquellos trabajadores. La clave aquí consistía en el hoy tan mítico objetivo de la investigación realizada en lugares «de difícil acceso»: no evidenciar la propia identidad.

${ }^{10}$ Por la ley de Enseñanza de 1870 , se hace obligatoria la escolaridad infantil. Es él momento en el que aparecen las bien conocidas Escuelas Públicas inglesas, cuyos poco educativos métodos aparecen relatadas en no pocas novelas y recreadas en algunas películas. Sobre este asunto, Jones (1981): 192-195. 


\section{La clasificación}

Combinando las respuestas de los visitadores con datos del censo, Booth establece una tipología de ocho clases sociales diferentes en la población estudiada:

- La clase A. Es la clase más baja, formada por trabajadores ocasionales y semi-criminales.

- La clase B. Los muy pobres. Formada por los trabajadores eventuales, caracterizados por un estado de necesidad crónica, viviendo al límite de la subsistencia.

- Las clases C y D. Los pobres, aquéllos cuyas ganancias son pequeñas debido a la irregularidad del empleo y aquellos otros cuyo trabajo, aunque regular, está poco pagado.

- Las clases E y F. Los empleados regulares y la clase obrera bien pagada de todas las ocupaciones.

- Clases G y H. Las clases medias y todas aquellas sobre este nivel (Poverty Series II, 20, $c f$. Pfautz 1967: 51).

Junto con esta clasificación, Booth elabora una cartografía de la distribución de dichas clases por las calles y viviendas del área estudiada. A través de una serie de criterios herederos de los debates higienistas, Booth va distinguiendo entre los «los problemas de desorden frente a los problemas de pobreza», o «los problemas de los pobres, frente a los problemas de las verdaderas clases trabajadoras» (Topalov 1994: 218). En el núcleo de su análisis se encuentra el problema de la irregularidad del empleo, y su relación con los hábitos de las clases pobres.

Booth trata de identificar un sub-tipo de pobres, distinguidos por sus «cualidades morales», es decir por sus hábitos y actitudes hacia el trabajo y el ahorro. A este grupo Booth les concede el derecho recibir un trato digno en las políticas públicas, pues son factores ajenos a ellos los que los condenan a una existencia precaria. «Mis "pobres" pueden describirse como aquéllos que viven en una lucha continua por obtener lo necesario para vivir» (Poverty Series, vol. I, 33 cf. Pfautz 1967: 52). Recogiendo en parte lo que será la mirada reformadora, Booth entiende que la situación de estos pobres no dependía sólo de sus características individuales, sino que se debía también a causas externas a ellos mismos que sólo pueden ser solucionadas mediante la intervención política.

Por el contrario, la situación de los muy pobres (la dos clases más bajas en su clasificiación) es debida, según el autor, a una forma de vida caracterizada por la aversión a la regularidad en el trabajo, la falta de ahorro o el alcoholismo. Son aquellos trabajadores eventuales de baja cualificación que alimentan el círculo vicioso de empleos inestables, dificultando la situación de otros trabajadores. Booth adscribe a este grupo no sólo un problema de hábitos, sino ca- 
racterísticas biológicas ${ }^{11}$. Este residuo, considerado insalvable, debía ser reducido mediante aislamiento o dispersión, evitando su posible contacto con otras clases sociales.

«Esta clase de gente salvaje, semi-criminal tuvo su período dorado cuando distritos enteros de Londres estaban en su posesión. Lo que es mejora a nuestros ojos, es destrucción a los suyos. Su descontento es la medida de nuestro éxito (...). Los marginados son suficientemente conscientes de esto, y oponiéndose testarudamente a los esfuerzos de la filantropía o el orden, su instinto de autoconservación busca un santuario intocable donde puedan permanecer juntos, asegurados por la mutua protección de sus respectivos caracteres para lo peor. Esto es lo que debe ser impedido. Tan pronto como se hacen con una calle, esta es víctima de desorden y debe ser destruida. La destrucción de tal propiedad no implica ninguna pérdida general. Las casas en las que viven no tienen más que un valor negativo, y sólo su destrucción puede constituir un avance» (Poverty Series, I, 172-178, cf. Pfautz 1967: 187).

Con el fin de indagar aún más sobre las cuestiones de la irregularidad en el empleo, Booth desplaza su objetivo en otras investigaciones al ámbito de la actividad laboral. Así, a lo largo de la década de los ochenta y noventa, Booth dirigirá varias investigaciones al frente de la Royal Statistical Society con el fin de conocer la situación de los estibadores ${ }^{12}$. Mediante datos obtenidos de los propios empleadores y de listas organizadas por los comités de cada oficio, Booth calcula los porcentajes de trabajadores con empleo regular e irregular en cada muelle. Es el alto porcentaje de estos últimos lo que preocupa especialmente a Booth, quien consagró su vida a demostrar que el trabajo irregular era el centro de un círculo vicioso de pobreza y degradación moral.

El interés de Booth por la situación de los trabajadores no vendrá motivado en ningún caso de las demandas de estos mismos. Como él mismo reconoce, la cuestión del empleo intermitente no constituye para los trabajadores el eje de la cuestión ${ }^{13}$. La obsesión por la regularidad en el trabajo procede, en realidad, de las preocupaciones higienistas de Booth por la transformación de los hábitos de una gran parte de la clase trabajadora. La inculcación del aprecio al trabajo regular será para Booth un objetivo primordial de su política social hacia las clases más pobres. Con este fin, propone el diseño de diversas reglamentaciones que a la larga transformen los hábitos y la actual organización del trabajo. En esta línea, Booth plantea para los estibadores la creación de un sistema de listas semi-

1 Galton (1822-1911), perteneciente ya al movimiento eugenista a partir del que la estadística matemática recibe un impulso definitivo, se apoyó en la clasificación de Booth para elaborar una teoría sobre la «naturaleza» de dichas clases (McKenzie 1981: 16).

${ }^{12}$ Estos informes fueron presentados la sesión inagural de su presidencia en la Sociedad, en noviembre de 1892 .

13 «Estos hombres (...) ven en el empleo de una plantilla semanal permanente una forma de impedir la distribución libre del trabajo y de debilitar su poder de combinación» (Booth 1892: 551). 
cerradas, de las que se «expulse» (de nuevo) a aquel residuo incapaz de llevar una regularidad en el trabajo:

«El sistema iría así contra dos clases: aquellos que no desean trabajo regular, y aquellos que se acercan al muelle no porque se demande su servicio como estibadores, o porque hayan elegido esa profesión, sino simplemente porque en ese momento no hay trabajo en otra parte.» (...) «Se me preguntará que qué será de los hombres que sean desplazados por este sistema. La respuesta es que el cambio vendrá poco a poco, y será más eficiente cortando la provisión de ingresos de estos hombres que de ninguna otra forma. Entre aquellos cuyas fuentes de empleo se agotarían, hay muchos que ahora dependen de los ingresos de sus mujeres e hijos, y que trabajan sólo lo necesario para conseguir tabaco y bebida para sí mismos. Por estos no debe sentirse ninguna pena» (Booth 1892: 553, 554).

\section{Booth, paradigma de la transición entre dos formas de representar y tratar las cuestiones sociales}

Booth representa una figura a caballo entre los higienistas y los reformadores sociales de finales de siglo. Frente a los higienistas de los años treinta, preocupados por la amoralidad de los pobres en general, Booth distingue un grupo de pobres cuya situación se debe a circunstancias totalmente ajenas a su voluntad. Este tipo de argumentación que libera a los pobres de su culpa será el eje de las propuestas reformistas y solidaristas de finales de siglo. Por otra parte, los grupos más pobres son en el esquema clasificatorio de Booth aquellos incapaces de practicar el trabajo asiduo. En éstos, los hábitos adoptados voluntariamente se confunden con las condiciones biológicas para convertirles en todo caso en colectivos a eliminar. En sus propuestas de reclusión o envío a colonias resuena el asilo benthamita.

En cuanto a los procedimientos de obtención de datos, la encuesta de Booth participa también de dos prácticas. Por un lado, mantiene el procedimiento de recogida de datos que había caracterizado a las primeras encuestas anglosajonas del siglo diecinueve, llevadas a cabo por las sociedades de caridad y por las emergentes sociedades estadísticas. En ellas, una serie de informantes privilegiados - notables, autoridades o miembros de instituciones locales como la policía o la escuela respondían a las preguntas realizadas, dando su parecer sobre la zona que ellos conocían. La posición institucional de los informantes les otorgaba la consideración de «representantes» de un lugar, sin necesidad de interrogar directamente a los sujetos a estudiar. Por otro lado, la investigación de Booth resulta especialmente novedosa por su proceso taxonómico posterior, que es elaboración exclusiva del observador. La abstracta nomenclatura (A, B, C, D...) muestra este intento de crear una clasificación científica, separada de los discursos y taxonomías presentes en la vida diaria. 


\subsection{Caroll D. Wright y las primeras encuestas sobre el paro}

El primer intento conocido de elaborar una encuesta con vistas a obtener una estadística del paro es el llevado a cabo por Caroll D. Wright, director del Labor Bureau de Massachusetts en 1878. La propia elección de Wright al frente del Labor Bureau es significativa de lo que será su papel en el mismo. Las visiones reformistas y pro-obreristas de su anterior director, Francis K. Olivier, habían provocado la irritación de patronos y banqueros hasta el punto de pedir la supresión de la Oficina. Como salida a estas críticas, el gobernador Washburn decide sustituir a Olivier, poniendo en su lugar a Wright, antiguo senador republicano que cuenta con el apoyo de los sectores más conservadores (Keyssar 1992: 353).

En 1878, ante una fuerte controversia en torno a las cifras de obreros sin trabajo, Wright se propone elaborar una estadística del paro en el estado. Para ello, se apoya también en una serie de informantes privilegiados: los oficiales de policía y los inspectores de impuestos. A éstos Wright les envía un cuestionario con indicaciones muy claras: anotar sólo el número de obreros masculinos mayores de 18 años, que «realmente deseen tener un empleo». Esto excluía, por ejemplo, a aquellos que no estuvieran dispuestos a trabajar por un salario mas bajo. Frente a las estimaciones que hablaban de casi un cuarto de millón de obreros sin trabajo, Wright y sus colegas se felicitan al ver que sus cifras no dan más de 22000 parados.

La encuesta de Wright inaugura toda una serie de investigaciones sobre el paro llevadas a cabo en ciudades como Pittsburgh o Nueva York, y que siguen la práctica de la encuesta anglosajona: la selección de un informante privilegiado al que se considera en capacidad de representar adecuadamente la situación. De esta forma se realizan una serie de cuestionarios que tendrán que responder las autoridades locales o las sociedades de beneficencia.

Por otra parte, la encuesta de Wright es pionera en la elaboración de un cuestionario específico con vistas a medir el paro. Es el propio cuestionario el que permite a Wright construir la categoría de parado. El paro emerge así a partir de los criterios del técnico estadístico, y no del sujeto estudiado, ni de otros sujetos mediadores.

Si Booth había podido establecer unas líneas de separación entre los pobres y los muy pobres que probablemente resultaban ajenas a ambos grupos, Wright establece una clasificación de los parados que difícilmente puede ser reconocida a ojos de los obreros sin trabajo. En concreto, Wright hacía tabla rasa de un aspecto clave en el conflicto que enfrentaba a patronos y obreros, y que repercutía directamente sobre las formas de definir la falta de trabajo: la cuestión del salario.

La figura de Wright puede interpretarse como parte del proceso histórico de neturalizacion del conflicto social a través del papel del experto. Alentado por el director de la Oficina del Censo, Francis A. Walker, tratará de hacer del Labor Bureau una institución modelo en su «tratamiento científico» de los problemas del mundo social, independiente de las visiones "partisanas», tanto de las asociaciones de empresarios, como de trabajadores. Con ese objetivo, Wright in- 
troducirá una novedad hacia los años ochenta: el envío de agentes encargados de establecer un trato personal con los informantes con el fin de observar afirmaciones contradictorias (Phelps Brown y Browne 1963). Dicho procedimiento se institucionalizará al poco tiempo mediante el nombramiento de corresponsales locales en las capitales de provincia.

Sorprende saber hoy que esta novedad, que haría del Labor Bureau de Massachussetts un pionero, no fue tan bien recibida en su momento. En un estudio llevado a cabo por la inglesa Royal Commission of Labor sobre las nuevas formas de encuesta, el procedimiento de Wright será duramente enjuiciado. Giffen, director del Departamento de Estadística del Board of Trade, protestaba en estos términos ante la Comisión: «No hay una sola persona en este mundo en quien yo confiaría las investigaciones de la forma que lo están haciendo algunos de los agentes norteamericanos» (Royal Commission of Labor, Fourth Report, 189394, $c f$. Phelps Brown and Browne 1963: 281).

La discusión abierta entre el Labor Bureau y la Royal Comission es signo de la vertiente política involucrada en la adopción de nuevos métodos de recopilación de datos sobre el mundo social. Ante la ausencia de una legitimación propia por parte de los nuevos científicos y observadores, y ante la ausencia de procedimientos mecánicos reconocidos, la veracidad de los datos, su objetividad, se somete a un arduo debate en el que no siempre se aceptó bien la llegada de agentes delegados que sustituyeran el conocimiento de las distintas autoridades locales.

Algunos años después tendrá lugar la elaboración de los primeros cuestionarios para realizar a los individuos. Tal es el caso de la encuesta Rowntree (1899) y del Informe del Department of Labor de Estados Unidos (1892). Es en estos años cuando por primera vez se llevan a cabo entrevistas individuales que sustituyen las apreciaciones de los informantes o las instituciones locales. No obstante, dichos cuestionarios son distribuidos en un primer momento a través de los trabajadores sociales o los inspectores de higiene, los cuales a su vez preseleccionan diferentes barrios o calles en los cuales sospechan que puede haber parados (Topalov 1994: 276). Así pues, pese a la introducción de la figura del agente entrevistador, la idea de representatividad permanecerá durante muchos años marcada por la mediación de sujetos reconocidos con capacidad para presentar la información justa (Didier 2001).

\subsection{Breve nota sobre la postura de las organizaciones obreras frente a la encuesta}

La mayor parte de las asociaciones obreras se sumaron al discurso de la modernidad, es decir, a esa nueva fe por descubrir verdades sobre el mundo social mediante métodos adecuados. Ahora bien, para las organizaciones obreras el deseo de conocer la verdad de la cuestión social formaba parte de la la necesidad de denunciar una situación con vistas a restituir un agravio realizado sobre el colectivo. 
Como parte de este proyecto de conocer la verdad, algunas organizaciones obreras se integraron en las nuevas asociaciones para la promoción de las ciencias sociales. Una vez allí, sus representantes vieron con estupor la incipiente práctica de las encuestas sociales. En una discusión de la National Association for the Promotion of Social Sciences, el secretario de una Asociación de Obreros de Londres protestaba en estos términos:

«En lugar de dejarles hablar por sí mismas, las clases obreras son aquí discutidas como si se tratara de una nueva especie o de un animal en extinción» (Cf. Himmelfarb 1991: 26).

Y Samuel Gompers, líder del movimiento obrero norteamericano, había hecho un comentario parecido algunos años antes:

«Los trabajadores no son insectos para ser examinados por intelectuales a través de las lentes de un microscopio, o por una excursión sociológica por los barrios pobres» (Cf. Davis 1984: 104).

\section{LA ENCUESTA COMO JUICIO PÚBLICO}

\subsection{La «Información Oral» y las informaciones del Instituto de Reformas Sociales}

En España, por un Real Decreto del 10 diciembre 1883 se crea una Comisión para investigar la situación de las clases trabajadoras. Siguiendo las sugerencias del Primer Congreso de Sociología celebrado unos meses antes en Valencia, se propone la creación de comisiones locales encargadas de distribuir un cuestionario entre las asociaciones y personalidades relevantes en cada localidad «con objeto de estudiar todas las cuestiones que directamente interesan a la mejora o bienestar de las clases obreras, tanto agrícolas como industriales, y que afectan a las relaciones entre el capital y el trabajo». Los cinco gruesos volúmenes que forman la Información Oral y Escrita ${ }^{14}$ son hoy un documento de gran riqueza, del que se puede obtener no sólo una ilustración viva de la situación de la clase obrera, sino también del concepto de ciencia social que tienen los reformadores de la España de fin de siglo.

La Información mantiene el procedimiento ya iniciado en el Primer Congreso de Sociología. En él no se presentan los resultados de investigadores reconocidos (una figura aún inexistente), ni siquiera las opiniones de juristas, economistas o moralistas. La Información Oral es un acontecimiento en sí, en cuanto que su importancia no radica sólo en el documento producido, sino tam-

${ }^{14}$ Los volúmenes de la Información Oral yEscrita fueron publicados entre 1889 a 1893. Con motivo del centenario de dicha obra el Ministerio de Trabajo y Seguridad Social publicó una reedición de los mismos con un estudio introductorio de Santiago Castillo (1985). 
bién en el momento de producción del mismo. En un proceso que dura varios días, se congregarán en el Aula Magna de la Universidad de Madrid representantes del capital, y de la pequeña burguesía ilustrada (los miembros de las Sociedades de Amigos del País, y los nuevos Ateneos), los cuales compartirán el espacio con representantes del trabajo, así como con jueces, miembros del clero y oficiales del gobierno.

El número de trabajadores excluido es amplio: sólo aquellos organizados como grupo participan. Muchas asociaciones, en cualquier caso, optan por no acudir: la bakuninista Federación de Trabajadores de la Región Española rechaza la invitación alegando que «el verdadero congreso ha tenido lugar ya en Valencia», refiriéndose al propio encuentro de las federaciones obreras que había tenido lugar pocos meses antes. La dura represión de las movilizaciones en los meses anteriores, y la actitud que parecía dominar entre los patronos hace que las asociaciones obreras desconfíen de la propuesta.

Cierto es que las comisiones locales están compuestas por cincuenta miembros, de los cuales sólo diez son obreros. No obstante, el objetivo principal de la Información Oral será precisamente interrogar y oír a los representantes obreros. Así pues, el Art. 10 para la formación de las comisiones locales, señala que el medio de información empleado por las comisiones para contestar dicho cuestionario será: «la prensa, las personas que se hayan ocupado de estas cuestiones, las compañías, círculos o ateneos que por razón de su fin puedan suministrar datos, y las asociaciones de obreros, a las cuales es deber de las comisiones oír en primer término» ${ }^{15}$. Es aquí donde radica la novedad de este procedimiento, que lo diferencia de las prácticas de los selected commitees: Los llamados a relatar el hecho social son fundamentalmente las organizaciones obreras. Son ellas las consideradas representantes legítimas para definir tal situacion.

Una decisión que sin duda viene marcada por el contexto: Un fuerte conflicto social, frente al que los reformadores sociales ven como única solución posible la integración de las organizaciones obreras en las instituciones del Estado. El Ministerio de Gobernación reconoce la importancia de este proceso como la única solución inmediata al conflicto político de esos años:

«No era posible prolongar esta situación sin menoscabo de la paz pública. Numerosos síntomas revelan que las clases obreras sienten el vivo estímulo de necesidades que remediar (...). Más estrechas parecen todavía las obligaciones del Gobierno, y con prontitud mayor debe atender a ellas, cuando una gran parte, acaso la más considerable de la clase obrera, reunida en el Congreso sociológico de Valencia, ha dado recientes y magníficas pruebas de amor a la legalidad y de confianza en los medios de la libre asociación individual, reclamando la intervención del gobierno tan sólo para remover los obstáculos que a su acción se oponen y para alcanzar aquellas facilidades que todo ciudadano tiene derecho a exigir y todo el que gobierna está obligado a otorgar siempre,

15 Instrucción pra las comisiones locales y provinciales encargadas de practicar una información sobre el estado y necesidades de la clase obrera. Art. 10. 
pero mucho más cuando los piden los menos favorecidos en el goce de las ventajas sociales» ${ }^{16}$.

El gobierno busca una forma de solución al conflicto mediante la integración de las asociaciones en las instituciones. Sin embargo, tal integración es imposible dado el funcionamiento de dichas instituciones y de las prácticas existentes en el ámbito local. Los reformadores sociales, que no sólo tienen en proyecto apaciguar a la clase obrera, sino también de construir una estructura que puentee las redes de patronazgo, buscarán canales alternativos que permitan la participación de las organizaciones obreras en los procesos políticos del Estado. La Información Oral puede interpretarse como uno de estos esfuerzos. Así, entre los meses de octubre y noviembre de 1884, se congregan en el Aula Magna cerca de 70.000 trabajadores en representación de todo tipo de asociaciones profesionales, de resistencia y de seguros mutuos.

\subsection{Un procedimiento peculiar de obtención de datos}

La invitación a las asociaciones obreras a participar en el Congreso de Sociología es una medida cuya dimensión política no se nos puede escapar, especialmente si tenemos en cuenta la posición que ocupaban las asociaciones obreras en ese momento. La trascendencia de tal acto es comparable a si en el próximo congreso de la Asociación Española de Sociología se invitara a colectivos de trabajadoras sexuales y personas sin techo a discutir sobre sus posibles «problemas» en lugar de que a lo máximo algún investigador simpatizante traduzca a la audiencia académica lo que supuestamente éstos son.

Del largo cuestionario de 194 preguntas, las asociaciones y corporaciones tienen la posibilidad de seleccionar aquéllas que consideren más relevantes para su posterior exposición pública. Estas preguntas son abiertas y dan así la posibilidad de elaborar todo un discurso. Siguiendo una lógica semejante a un juicio, pero también a una sesión parlamentaria, el presidente correspondiente de la Comisión llama cada día a dos o tres representantes de las asociaciones para que expongan públicamente su respuesta a algunas de las cuestiones tratadas. Ante una audiencia mayoritariamente simpatizante, los representantes obreros tienen así la oportunidad de denunciar su situación sin cortapisas a un lenguaje emotivo que expresa con viveza la misma. El tono dramático se combina con momentos cómicos formando en conjunto el ritual de una escena, cuyo efecto terapéutico es evidente.

La Información Oral procede, por un lado, de los deseos de los republicanos y algunos reformadores sociales de inquirir en la situación de las clases obreras con objeto de cambiar ésta y aproximarles a la condición de ciudadano, Por otro, es una vía intermedia de acercamiento de los colectivos obreros a la legalidad y

${ }^{16}$ Real Decreto de creación de una Comisión de estudio de cuestiones obreras. Gaceta 10 diciembre de 1883 (publicado en la mencionada edición). 
al proceso normativo-institucional, que no supone, sin embargo, su participación inmediata y de pleno derecho en las instituciones existentes. Es, por tanto, una vía abierta a un nuevo agente político, que no tiene cabida en las formas existentes de pensar y articular la participación política.

\subsection{El paro en la Información Oral}

Las cuestiones de la Información giran en torno a las condiciones de vida y trabajo de las clases obreras, estableciendo eventualmente comparaciones con otras clases. El tema de la falta de trabajo es también tratado como parte del conjunto de situaciones que afectan a la clase obrera, y no a un grupo separado de la misma. A lo largo del proceso interrogatorio, algunos representantes de obreros elaboran una taxonomía en torno a la problemática del paro. Pero dicha taxonomía dista mucho de la categorización del parado en el cuestionario elaborado por Wright para el Bureau de Massachussetts, y sin duda, de la visión de Booth respecto al trabajo irregular. Así, Hipólito, de la Sociedad de Socorros a Parados, distinguía las siguientes situaciones de paro:

«Yo divido a los obreros parados en cuatro grupos:

$1 .^{\circ}$ los parados por falta de trabajo.

$2 .^{\circ}$ Los parados porque están enfermos.

3. Los parados por causas de su ancianidad.

4. ${ }^{\circ}$ Los parados por dignidad, o sea, los que abandonan el trabajo por dignidad. El obrero que ha estado viente o treinta años trabajando para ganar un pedazo de pan que llevar a la boca, ese obrero que se encuentra en un estado en que necesita ganar más porque tiene que atender a su familia y quizás a sus padres ancianos, ese obrero llega al taller, y al ver que por lo mismo que ya está fatigado se le quiere exprimir más, llega una ocasión en que dice: muerto por mil, muerto por mil y quinientos; no trabajo más. Sale a la calle, levanta con arrogancia su vista, pero bien pronto la tiene que bajar, porque se encuentra con la realidad, y la realidad es la pérdida del trabajo. El estar parado lleva consigo una tristeza, que muchas veces se convierte en llanto $(. ..) ; \mathrm{A}$ dónde va el hombre que ha dejado el trabajo por dignidad? Porque, señores, yo no comprendo cómo hay escritores que venden su conciencia por dinero. (...) Yo les aseguro desde aquí que si, por ejemplo, hoy somos 20.000 obreros los parados en Madrid, antes de ocho años hemos de ser 60.000 y no ya en Madrid, sino en todas partes donde haya obreros. Ved si no lo que ocurre en Francia, y yo añado que cuanto mayor sea el criterio de las clases trabajadoras, cuanto más lata sea su instrucción, más numerosas han de ser las crisis (IOE, vol. I: 54, 55).

Interrogado, el presidente de la sociedad de socorros a parados no podía separar la situación del parado de la relación de poder establecida en torno al trabajo. Su taxonomía creaba así categorías regidas por la lógica opuesta a las categorías de Booth o de Wright. 
La Información puede sin duda sorprendernos hoy en día. Un procedimiento novedoso para el momento, que tiene su equivalente en una encuesta francesa realizada en los mismos años ${ }^{17}$. Sin embargo, en el país de Comte y Durkheim, aquélla quedó en caso aislado. En España en cambio, la propuesta de la Información animará la puesta en marcha de una práctica regular por parte de los reformadores que trabajan en el Instituto de Reformas Sociales entre 19031923. Promovido por Canalejas, el Instituto es el primer intento de creación de un cuerpo de profesionales encargados de analizar la situación de las clases obreras. Su centro en Madrid está cuenta con un porcentaje importante de su personal procedente de la universidad. Entre estos, dos figuras le imprimen especial carácter: Adolfo Posada y Adolfo Buylla. Republicanos y krausistas, procedentes ambos del llamado Círculo de Oviedo, los «Adolfos» son las personas más activas del Instituto. Estando al corriente de los cambios institucionales llevados a cabo por los reformadores de otros países, y de los debates existentes en el campo de la reforma social, los «Adolfos» son el ejemplo clave de la figura del reformador social como canal de transmisión e intermediación: un sujeto inserto en las redes internacionales, en esa cuasi comunidad epistémica, y al mismo tiempo, buen conocedor de las circunstancias concretas de su propio país.

Adolfo Buylla, profesor de Economía Política en la Facultad de Oviedo, es nombrado presidente de la Sección de Estadística (la Sección Tercera) del Instituto de Reformas Sociales. Entre 1903 y 1923, esta sección presenta regularmente datos sobre huelgas, precios de los principales productos y salarios. Al mismo tiempo, la Sección Tercera llevará a cabo un gran esfuerzo por elaborar lo que Buylla considera el principal instrumento de investigación: las informaciones. Realizadas en una zona concreta y casi siempre tras el estallido de un enfrentamiento social, recaban testimonios y opiniones de las partes en conflicto (patronos industriales o grandes propietarios agrícolas y organizaciones de trabajadores), así como de periodistas y otros observadores. El objetivo fundamental de estas informaciones consiste en trascender los informes oficiales sobre la situación en dicha localidad o industria. Para lograr tal objetivo, los encargados buscan ante todo entrevistarse con las organizaciones obreras con el fin de conocer el desarrollo de los acontecimientos y las circunstancias en que se encuentran ${ }^{18}$.

El uso del término información como sinónimo de encuesta social es propiciado por el propio Buylla. En su traducción del Tratado de Estadística de Filippo Virgilii (1898), emplea el término información como equivalente al italiano inchiesta [encuesta]:

17 La encuesta francesa, publicada entre los años 1884 y 1885 , se lleva a cabo igualmente mediante un proceso oral, a partir de un cuestionario dirigido, entre otras, a las organizaciones sindicales. Un análisis de la misma en Simiand (1934) y Perrot (1974).

${ }^{18}$ La adopción del punto de vista de las asociaciones obreras resulta especialmente evidente en los pasajes de la Información Agraria en ambas Castillas, elaborada por Buylla en 1904, y parcialmente también en la Información sobre el problema agrario en la provincia de Córdoba de 1919 , en la que también interviene el autor. Sobre las informaciones y el papel del Instituto de Reformas Sociales en la historia de la sociología española, Zarco (1999). 
«Es una investigación de uno o más elementos de un territorio, y puede servir también de comprobación o verificación o corrección de los hechos observados. Puede emplearse con cierta continuidad o periodicidad; pero en general se acude a ella cuando se quiere apoyar en datos estadísticos un proyecto de ley, o se pretende demostrar la necesidad de una medida administrativa. Pertenece esta forma rigurosamente a la recolección ocasional. Al hablar de informaciones nos referimos a las que versan sobre asuntos sociales, por ejemplo, la de las condiciones de la agricultura italiana, sobre las huelgas, sobre la circulación monetaria, o sobre los institutos de beneficencia».

\section{Aquí Buylla añadía en una nota a pie de página:}

«Entre las informaciones, que en cierto sentido cabe considerar como estadísticas en España, debemos notar dos de marcado carácter social: una relativa a la emigración en Julio de 1881 , y la otra promovida por la Comisión de Reformas Sociales para conocer la situación de las clases obreras, realizada en Abril de 1884» (Virgilii 1898: 44) ${ }^{19}$.

Sorprende que Buylla erigiera la información en el método por excelencia de observación de lo social; más aún cuando el autor conocía los nuevos métodos estadísticos aplicados a la investigación social ${ }^{20}$. Una interpretación plausible del camino tomado por Buylla es que este republicano de filiación socialista, y perteneciente a los círculos krausistas ${ }^{21}$, nunca habría aceptado la elaboración de cuestionarios sistemáticos y cerrados. Tal y como él mismo defendió la información:

«No se trata aquí de teorizar acerca del carácter y significancia de este procedimiento, genuinamente sociológico, que es estadística, historia y monografía, todo en uno. Porque [la información] anima y hace vivo el dato numérico por la expresión de todos aquellos aspectos del individuo y la persona social que escapan a la rigidez. Es más real ya que la evidencia son los actores mismos, y es un instrumento estable de certidumbre, en cuanto la omnilateralidad del testimonio implica la audiencia de todos los intereses y la consideración de todas las tendencias» (Buylla 1904).

${ }^{19}$ Manual de Estadística de Filippo Virgilii. Segunda edición (1898), traducido y con notas añadidas por Adolfo Buylla en. Madrid, España Moderna, pág. 44.

20 La amplia bibliografía tanto extranjera como española añadida a la traducción del Tratado de Virgilii demuestra que Buylla manejaba las nuevas publicaciones sobre el asunto.

${ }^{21}$ La trayectoria intelectual y política de Buylla nos señala la vertiente de la reforma social en España, concebida como democratización y no como represión. Catedrático de Economía Política, Buylla será muy crítico con el utilitarismo y la economía liberal. Asimismo, Buylla perseguirá toda su vida poner fin a la pobreza, pero sobre todo, al tratamiento estigmatizante que el pobre recibe de la beneficencia. Al igual que tantos otros reformadores sociales de su tiempo, Buylla verá en la asociación obrera, la salida fundamental de esa forma de dependencia. Así, en 1894, siendo concejal en el Ayuntamiento de Oviedo, propondrá la Creación de una comisión de protección para las clases menesterosas diseñada para fomentar el espíritu de asociación entre las éstas y las clases trabajadoras (Crespo Carbonero 1995: 127). 
Para Buylla la finalidad de la investigación sociológica es sobre todo proporcionar un juicio justo, adecuado, sobre una situación. Ello supone escuchar a las distintas partes, y muy especialmente, a aquellas hasta ahora silenciadas: las clases trabajadoras. La idea de investigación social de Buylla está en realidad marcada por su republicanismo y la influencia del krausismo. El republicanismo buscaba ante todo la tranformación de las clases trabajadoras en ciudadanos activos, es decir, sujetos educados (aunque en la cultura dominante) y con reconocida capacidad de autonomía por las instituciones. El krausismo, por otra parte, era un enfoque ecléctico desarrollado por una serie de intelectuales españoles unidos en torno a la Institución Libre de Enseñanza ${ }^{22}$ que compartían, entre otras cosas, un distanciamiento del positivismo defendido por los círculos internacionales de reformadores sociales de finales de siglo. Los krausistas, más cercanos quizás al idealismo romántico, tienen una idea de progreso entendida como educación del espíritu. Esta idea les lleva a reflexionar sobre las condiciones para regenerar el país y sobre todo para la formación de ciudadanos. Al mismo tiempo, los krausistas tienen una concepción organicista de la vida social según la cual la armonía sólo puede lograrse mediante la cooperación de las distintas partes representadas por sus distintas asociaciones.

Teniendo en mente el trasfondo intelectual en el que se mueven Buylla o Posada, resulta más claro por qué no podía tener sentido para ellos un enfoque de la cuestión social a través de unas lentes científicas, que objetivasen desde fuera cuál era el estado de las clases trabajadoras. Pues, por un lado, uno de los objetivos fundamentales de los reformadores krausistas será precisamente fomentar la participación de las asociaciones de los trabajadores en la vida política y en sus instituciones. Por otro lado, Buylla, como se deduce de su propia definición de la información, entiende que el justo conocimiento de lo social sólo puede resultar de una ampliación de la perspectiva, en la que se integre el punto de vista del sujeto hasta ahora desconocido o silenciado.

El Instituto de Reformas Sociales se convierte en un lugar de investigación, propuesta de legislación social y mediación. Todos estos proyectos se enteremezclan en las informaciones, las cuales adquieren el formato de la recogida de testimonios en un lugar donde ha estallado el conflicto. La exactitud del dato se identifica con la justicia derivada de la escucha de todas las partes, y en especial de aquellas hasta ahora condenadas al silencio: los trabajadores.

${ }^{22}$ Se ha escrito mucho sobre el krausismo, a veces asumiendo que se trataba de un corpus de pensamiento, de la misma forma que se puede hablar del hegelianismo o del marxismo. En realidad el krausismo, es un término más bien accidental, que se debe al viaje de Sanz del Río a Alemania, quien recibió allí clases de Krause, un profesor de universidad, que al menos hoy en día no ha dejado ninguna huella en los historiadores del pensamiento alemán. El krausismo en cambio sí dejo una amplia huella en España, porque con él se denota al grupo de intelectuales más próximo al reformismo social en sus vertiente aperturista y democratizadora, y a todas las actividades desarrolladas por la Institución Libre de Ensañanza que abrieron un camino clave hasta los últimos años de la II República. Los krausistas y en especial la Institución Libre de Enseñanza serían así destestados por las corrientes principales del pensamiento conservador que abrieron paso al régimen franquista. Así, en los años veinte Ramiro de Maeztu situaba la causa de los males de España en la ILE (Botti 1992).

EMPIRIA. Revista de Metodología de Ciencias Sociales. N. ${ }^{\circ}$, enero-junio, 2005, pp. 115-141. 
Esta visión de la ciencia social era en realidad la que reclamaban las organizaciones de trabajadores, pese a que en muchos casos desconfiaran de las posibilidades del Instituto de Reformas Sociales. Así, el anarquista Anselmo Lorenzo, enfatizaba en un artículo la importancia de la «verdad sociológica, extendida por la Internacional» (Lorenzo (1893). Acercándose a la noción marxista de conciencia, Lorenzo identificaba la verdadera sociología con los movimientos sociales. Así, en otro artículo publicado 1895, tras pasar revista a las diferentes huelgas y movilizaciones que estaban teniendo lugar Europa en esos años, Lorenzo resumía con estas palabras la situación:

«Como una consecuencia lógica del desarrollo de la sociología en el último período, hay un flujo en la opinión pública que corresponde con las actuales necesidades y preocupaciones de la sociedad». (...) «Se busca la ley que gobierna los hechos, y de su descubrimiento emerge la consecuente acción colectiva» (Lorenzo 1895).

Frente a esta forma de entender la sociología, Lorenzo desconfía profundamente de la actividad académica. Esta desconfianza iba también para aquellos que se presentaban como portavoces del modelo de nueva ciencia social:

«La Universidad de Chicago ha acordado iniciar una nueva revista, la American Review of Sociology con el supuesto objetivo de combatir la pseudosociología, separándose de la utopía social y la doctrina cerrada. Bajo nuestro punto de vista, esta revista representa, aunque de una forma encubierta, un intento por la burguesía de eliminar toda posible influencia socialista en los trabajadores»(Lorenzo 1895: 87).

Los reformadores sociales de impronta socialista, como Buylla o Posada se negaron también a separar el acto de observación de su sentido político. La sociología, significa para este grupo de reformadores salir del aislamiento impuesto por la administración, así como de la soledad y la autoreferencialidad de la universidad y de la academia jurídica ${ }^{23}$. Entre 1903 y 1923, los miembros del Instituto produjeron una rica serie de informaciones sobre el trabajo en las minas,

${ }^{23}$ Las líneas de este proyecto, que nunca llegó a llevarse a cabo totalmente, pueden leerse en Buylla, Posada, Morote [1902] (1986). Los autores hablan de la necesidad de «desarrollar un sistema de informaciones que permita suplir la falta de la potencia convincente de los hechos en masa, por medio del examen circusntanciado de los diferentes aspectos de la materia. Un sistema en el que toman parte activa los mismos interesados aportando un verdadero juicio contradictorio, comunicando a la obra sabor y color de realidad que no se obtiene por modos tan acabados en la estadística al uso». Y añadían, como ya reconocía el sabio Rúmelin al decir hace bastantes años que «a las cifras es necesario abrirles la boca» (1902: 45). Observando instituciones análogas de otros países, les resultarán insuficientes los modelos de l'Office du Travail francés, o del Labor Bureau de Estados Unidos por ser oficinas burocráticas, centradas casi exclusivamente en la información estadística y sin capacidad de legislación (1902: 150-162). El sueño de estos reformadores es que el Instituto de Trabajo se convirtiera también en un órgano de legislación a partir de la participación de representantes patronos y obreros. 
sobre la situación de obreros agrícolas en varias regiones o sobre la industria doméstica. En ellas, el encuentro con las organizaciones obreras se considera una condición fundamental para conocer una situación, pero además un paso necesario en su proyecto democratizador. La preocupación del grupo de reformadores sociales vinculados a la Institución Libre de Enseñanza por la exclusión de las clases trabajadoras en los procesos políticos, así como por sus condiciones de vida, convierte las informaciones en un instrumento de denuncia, que anima un todo un proceso legislativo y de cambio institucional.

Pero antes de que este proceso pudiera implementarse, soplaban ya nuevos aires. La llegada de la dictadura de Primo de Rivera creó un clima en las instituciones favorable a la introducción de una retórica de cientificidad, entendida como un conjunto de reglas rígidas. Ideas sobre la organización técnica de la investigación social, coetáneas a las nuevas ideas sobre la organización científica del trabajo, cuadraron perfectamente con la introducción del ejército en gran número de instituciones. La modernidad se ideaba así con sus trazos más racionalizadores.

El Instituto de Reformas Sociales fue suprimido en 1923 y sus competencias pasarán al Ministerio de Trabajo. Es su seno se crea un nuevo departamento encargado de la elaboración de todo tipo de estadísticas sociales y del trabajo e integrado por una nueva generación de técnicos estadísticos salidos de la universidad y de las escuelas técnicas. Dicha generación se caracteriza por un sentimiento de fidelidad hacia su propia profesión que los mantendrá alejados de las preocupaciones más mundanas de la anterior generación de reformadores sociales ${ }^{24}$.

Entre sus realizaciones se encuentra la introducción de la estadística matemática en España. Por otra parte, su labor desplaza a los anteriores miembros del Instituto de Reformas Sociales, que serán de ahora en adelante oídos sólo en ocasiones muy concretas. Así se expresaban los nuevos miembros del Cuerpo Facultativo de Estadística en el contexto de un proyecto de estadísticas del paro:

«Los antiguos delegados de Estadística del Instituto de Reformas Sociales recogen o poseen documentación y noticias sobre estas materias. Su cooperación puede ser útil en el aspecto cualitativo más que en el cuantitativo porque su contacto con las realidades obreras y su conocimiento de las peculiaridades locales permitirá obtener de ellos la aclaración y la explicación de las cifras, especialmente cuando se presenten irregularidades o anomalías en la evolución del fenómeno» (Guichot y Ruiz Almansa 1925: 46).

\section{CONCLUSIONES}

El paro es un concepto que surge contemporáneamente al nacimiento de las ciencias sociales y la estadística como procedimientos para establecer un juicio

${ }^{24}$ Se pueden revisar las biografías de algunos de estos nuevos técnicos estadísticos (Arribas Macho 2004, García Álvarez 1981, Martínez y Rey 2003) para comprender la ruptura que marca esta generación con los pensadores del Círculo de Oviedo y de la Institución Libre de Enseñanza. 
adecuado sobre los hechos del mundo social. Pero las prácticas de dichas disciplinas tardarán mucho tiempo en asentarse. Así pues, los primeros documentos sobre el paro reflejan unas nociones de investigación y representatividad muy diversas, de las cuales resulta también una variedad de formas de categorizar la idea general de «falta de trabajo».

La encuesta anglosajona se caracteriza por el fuerte papel del delegado y el observador. Estos acabarán con el tiempo sustituyendo a la autoridad local que durante los siglos dieciocho y diecinueve había sido reconocida con la capacidad para emitir un juicio adecuado sobre la situación social del lugar que representaba. Ante la pérdida de legitimidad de tales autoridades el sociólogo y el estadístico se van abriendo camino, reconocidos en su caso no tanto por quienes son, sino más bien por lo que hacen.

Las informaciones realizadas en España se caracterizan, por el contrario, por el fuerte papel del sujeto observado. Es él, en este caso la clase obrera, el informante privilegiado al que interesa oír sobre su situación. Frente a lecturas sobre el atraso intelectual español, la interpretación alternativa aquí propuesta es que tal procedimiento respondía a un intento democratizador por parte de los reformadores sociales.

La historia sin embargo dio la razón a la encuesta anglosajona, la cual adquiere todo su esplendor a partir de los años treinta y sobre todo tras la Segunda Guerra Mundial ${ }^{25}$. Pero la elección no era inocua: las formas de investigación del mundo social tienen consecuencias directas sobre la definición del mismo. El caso de las primeras encuestas sobre el paro evidencia las distintas definiciones que puede adoptar una noción como la falta de trabajo en función de los procedimientos de interrogación sobre la misma.

${ }^{25}$ Tras la Segunda Guerra Mundial culmina en Estados Unidos la versión más tecnocrática de la encuesta estadística. Este proceso es analizado en Pollack (1979). Como vía intermedia quedarán las distintas formas de investigación cualitativa, en las que en cualquier caso y quizás a excepción del grupo de discusión, la distancia epistemológica provocada por una determinada concepción de la ciencia social no tendrá ya vía de retorno. 


\section{REFERENCIAS}

ARribas (2002): Introducción al texto de A. Bowley «La aplicación del muestreo a los problemas económicos y sociológicos», Empiria, n. ${ }^{\circ}$ 5, pp. 195-204.

BooTh, C (1892): «The Inaugural Address of Charles Booth, Esq., President of the Royal Statistical Society. Session, 1892-93», Journal of the Royal Statistical Society, vol. 55, n. ${ }^{4}$ (Dec. 1892), pp. 521-557.

BotTI, A (1992): El cielo y el dinero. El nacionalcatolicismo en España (1881-1975). Madrid, Alianza Universidad.

Bulmer, M. (ed.) (1985): Essays on the History of British Sociological Research. Cambridge, CUP, «Introduction».

BULMER, Bales and SKLAR (1991): The social survey in historical perspective, 1880-1940. Cambrige, CUP.

Buylla, POSADA, MOROTE [1902] (1986): El Instituto de Trabajo. Datos para la historia de la Reforma Social en España. Madrid, Ministerio de Trabajo y Seguridad Social.

BUYLLA (1904): Memoria acerca de la Información agraria en ambas Castillas. Madrid, Imprenta Sucesora de Minuesa de los Ríos.

CAMARERO, L. (2001): «Los soportes de la encuesta: la infancia de los métodos representativos». Metodología de Encuestas, vol. 3, n. ${ }^{\circ}$ 2, pp. 163-181.

CRESPo CARbonero, J. A. (1995): Teoría económica, estructura legal y educación popular para la reforma social en Adolfo A. Buylla. Tesis de doctorado. Universidad Autónoma de Madrid.

CASTILlo, S. (ed.): Reformas Sociales. Información Oral y escrita publicada de 1889 A 1893. Madrid, Ministerio de Trabajo y Seguridad Social (reedición de la publicada por la imprenta Minuesa de los Ríos).

Chandler, J.; Davidson, A. I. y Harootunian, A. I. (1991): Questions of evidence. Proof, Practice, and Persuasion across the Disciplines. University of Chicago Press.

Cullen, M. J. (1975): The Statistical Movement in Early Victorian Britain. The Foundations of Empirical Sociological Research. New York. Harvester Press.

Davis, A. F. [1967] (1984): Spearheads for Reform. The Social Settlements and the Progressive Movement, 1890-1914. New Brunswick.

Desrosières, A. (1993): «La politique des grands nombres». Histoire de la raison statistique. Paris, la découverte. Edición en castellano (2004).

DIDIER, E. (2001): De l'échatillon à la population. Sociologie de la géneralisation par sondage aux États-Unis avant la Seconde Guerre Mondiale. Thèse de doctorat. École des Mines de Paris.

Guichot, J y Ruiz Almansa, J (1925): El paro involuntario y la estadística del mismo. Madrid, Impresos de Tejada, 46.

Himmelfarb, G. (1983): The Idea of Poverty. England in the Early Industrial Age. New York, Knofp.

- (1991): Poverty and Compassion. The Moral Imagination of the Late Victorians. New York, Alfred A. Knopf.

JONES, G. S. (1971): Outcast London

- (1981): Languages of Class. Studies in English Working Class History. Cambridge, CUP.

KeYsSAR, A. (1986): Out of Work. The first century of unemployment in Massachusetts. Cambridge, CUP.

LECERF: «Les Conférences internationales pour la lutte contre le chômage au début du siècle», Mil Neuf Cent, $\mathbf{n} .^{\circ}$ 7, 1989. 
LORENZO, A. (1893): «La cuestión social», Ciencia Social, n. ${ }^{\circ} 1$.

- (1895): «Movimiento Social», Ciencia Social, n. ${ }^{\circ} 3$, pp. 27-29.

- (1895): «Noticias. La American Review of Sociology», Ciencia Social, n. ${ }^{\circ} 3$, p. 87.

MaCKenZIE, D. A. (1981): Statistics in Britain, 1865-1930. The Social Construction of Scientific Knowledge. Edinburgh University Press.

MASH, C, (1985): «Informants, respondents and citizens», en Bulmer, pp. 206-227.

Pfautz, Harold W. (1967): Charles Booth on the City. Physical Pattern and Social Structure. Chicago, University of Chicago Press.

Phelps Brown, E. H. and Browne, M. H. (1963): «Caroll D. Wright and the Development of British Labour Statistics», Economica, August, pp. 277-286.

Perrot, M. (1974): «Comment les ouvriers parisiens voyaeint la crise d'après l'enquête parlementaire de 1884», Conjoncture Économique. Structures Sociales. Hommage à Ernest Labrousse. Paris, Mouton, pp. 187-200.

PollaCK, M. (1979): «Paul Lazarsfeld, fondateur d'une multinationale scientifique», Actes de la Recherche en sciences sociales, 25, fév: 45-60.

PORTER, T. (1995): Trust in Numbers. The pursuit of objectivity in science and public life. Chicago, University of Chicago Press.

RUESCHMEYER, D. and SKOCPOL, T. (1996): States, Social Knowledge and the Origins of Modern Social Policies. Princeton, PUP.

SAlaIS, BAVERED, REYNAUd (1986): L'invention du chômage. Histoire et transformations d'une catégorie en France des années 1890 aux années 1980. Paris, Presses Universitaires de France.

SimiAnd (1934): «Une enquête oubliée sur une grande crise méconnue», Melanges d'économie politique et sociale offerts à Edgar Milhaud. Paris, Presses Universitaires.

Topalov, C. (1991): Topalov, C «La ville, "terre inconnue". L'enquête de Charles Booth et le peuple de Londres, 1886-1991», Genèses, 5, septembre: 5-34.

- (1994): Naissance du chômeur, 1880-1910. Paris, Albin Michel.

VIRGILII, F. (1898): Manual de Estadística. Segunda edición, traducido y con notas añadidas por Adolfo Buylla. Madrid, España Moderna.

WERnER, M et ZimmermanN, B. (2003): «Penser l'histoire croisée: entre empirie et réfliexivité» Annales $H S S$, janv-février, pp. 7-36.

ZARCO, J. (1999): «Notas sobre el instituto de reformas sociales ylas tres historias de la sociología española», Revista Española de Investigaciones Sociológicas, 86, pp. 129-152.

ZIMMERMANN, B. (2001): La constitution du chômage en Allemagne. Entre professions et territoires. Paris, Éditions de la Maison des sciences de l'homme. 


\title{
RESUMEN
}

El artículo compara dos tipos de investigación social desarrolladas a finales del siglo diecinueve: la encuesta angosajona y las informaciones españolas. Situándose en un tema concreto - el paro- el artículo analiza éstas como diferentes formas de establecer objetividad sobre el mismo, al tiempo que insiste en la cuestión de la vertiente política de las prácticas científicas.

\section{PALABA ClaVE}

Representatividad, encuesta, historia de la estadística.

\begin{abstract}
The article compares two types of social inquiry developed at the end of the nineteenth century: the Anglo-Saxon survey and the Spanish informaciones. By focusing on a concrete issue - unemployment - the article analyses them as different forms of establishing objectivity about it and it thus empahsises the political character of scientific practices.
\end{abstract}

\section{KEY WORDS}

Representative, survey, history of stadistics. 